\title{
Learning from Preferences and Selected Multimodal Features of Players
}

\author{
Georgios N. Yannakakis \\ Center for Computer Games Research \\ IT University of Copenhagen \\ Rued Langgaards Vej 7, DK-2300 \\ yannakakis@itu.dk
}

\begin{abstract}
The influence of multimodal sources of input data to the construction of accurate computational models of user preferences is investigated in this paper. The case study presented explores player entertainment preferences of physical game variants incorporating two data modalities. The main findings of the paper reveal the benefit of multiple modalities of input data for the prediction of preferences and highlight the impact of feature selection on the construction of such models.
\end{abstract}

\section{Categories and Subject Descriptors}

I.2.1 [Artificial Intelligence]: Applications and Expert Systems-Games; I.2.6 [Artificial Intelligence]: Learning-Connectionism and neural nets; H.1.2 [Models and Principles]: User/Machine Systems-Human factors

\section{General Terms}

Design, Experimentation, Human Factors, Performance

\section{INTRODUCTION}

Rich forms of interaction can be designed when the preferences of a user of an interactive system are successfully modeled. Multimodal sources of input data can provide the necessary ground for constructing accurate computational user models in such systems. These models can be used for re-engineering the system and adjust internal controls during the interaction for maximizing the output value of the model function.

In this paper we investigate the impact of multimodality to the construction of user models (multinodality refers to the different modes of input data investigated in this paper). Multimodality is investigated through a physical interactive game and user models are built on entertainment ("fun") preferences of its players. Accurate models of user preferences have already been reported in the literature built on

Permission to make digital or hard copies of all or part of this work for personal or classroom use is granted without fee provided that copies are not made or distributed for profit or commercial advantage and that copies bear this notice and the full citation on the first page. To copy otherwise, to republish, to post on servers or to redistribute to lists, requires prior specific permission and/or a fee.

ICMI-MLMI'09, November 2-4, 2009, Cambridge, MA, USA.

Copyright 2009 ACM 978-1-60558-772-1/09/11 ...\$10.00. single modalities of gameplay interaction [11] and physiological signal data [8]. This paper extends the existing knowledge on user models of entertainment preference by building models of the user on the unified set of physiological and gameplay interaction data. Artificial neural networks (ANNs) are constructed using artificial evolution to learn the mapping between individual features extracted and user preferences of entertainment. For this purpose two feature selection mechanisms are designed to choose the appropriate input vector for the model: sequential feature selection (SFS) and perceptron feature selection (PFS).

Results show that models of user preferences built on multimodal input data are more accurate than the corresponding models built on unimodal data verifying the underlined assumption. The highest performing model achieves a crossvalidation performance of $83.33 \%$ on unseen data. Moreover, SFS proves to be more efficient than PFS in searching for the appropriate subset of features towards constructing this model. Overall, SFS selects feature subsets for the ANN that generate higher performance than the corresponding subsets selected by PFS.

\section{EXPERIMENTAL PROCEDURE}

The procedure we follow for building accurate models of user preferences is illustrated in Figure 1. First, an experimental protocol is designed to elicit genuine emotional responses (preferences) from users of the interactive system [9]. Then data deriving from multiple modalities are collected through the experiment and statistical features are extracted. Given the expressed preferences and the statistical features, preference learning is applied for approximating the function between selected feature subsets - derived from efficient feature selection - and reported preferences. Accurate functions of that relationship define the required user models of preference.

In this paper we investigate the case study of interaction with physical activity games and expressed preferences of entertainment on these games. Gameplay interaction data (i.e. interaction via foot pressed tile events on the game platform) and physiological signal data define the two data modes examined in this paper. The merging of the data in a single model is investigated and the assumption of a more accurate model is evaluated using two feature selection schemes to select a features subset from the feature set (71 features in total) extracted from the data. The reader is referred to [8] for more details on the experimental protocol used. 


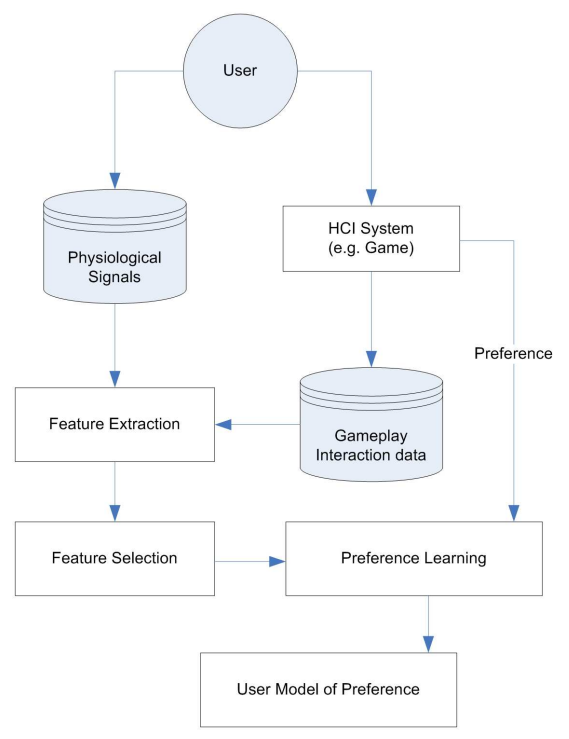

Figure 1: Methodology for constructing quantitative models of user preferences.

\section{PREFERENCE LEARNING}

The proposed approach to user modeling is based on selecting a minimal subset of individual features, on the basis of which is constructed a quantitative user model that predicts the subject's reported entertainment preferences. The assumption is that the entertainment value $y$ of a given game, which models the subject's internal response to playing the game, that is, how much "fun" it is, is an unknown function of individual features which a machine learning mechanism can learn [11].

Constraint satisfaction algorithms cannot solve the problem since the variable $y$ under the constraint $y_{A}>y_{B}$ for any two given games $A$ and $B$ has no specific domain values. Likewise, any machine learning which is based on learning a target output is inapplicable since target outputs are unknown. Preference learning [1] is the only applicable type of machine learning for this constrained classification problem. There are several techniques that learn from a set of pairwise preferences such as algorithms based on support vector machines, gaussian processes and evolving ANNs.

\subsection{Evolving ANNs}

Feedforward multilayered neural networks are employed for learning the relation between the selected player features (ANN inputs) and the "entertainment value" (ANN output) of a game. Since there are no prescribed target outputs for the learning problem (i.e. no differentiable output error function), ANN training algorithms such as backpropagation are inapplicable. Learning is achieved through artificial evolution. The motivation behind the use of evolving ANNs is two-fold: a) the high level of subjectivity of human preferences and the noisy nature of input data require complex non-linear functions to be used; and b) evolving ANNs have shown performance advantages over other preference learning mechanisms in constructing models of user preference [8].

The sigmoid function is employed at each neuron of the ANN, the connection weights take values from -5 to 5 to match with input values that are normalized into $[0,1]$. The topology of the ANN is fixed; evolution adjusts only the connection weight values of the ANN. A generational genetic algorithm (GA) [3] is implemented, which uses a fitness function that measures the difference between the children's reported preferences and the relative magnitude of the corresponding model output values $y$. The ANN is itself evolved. The algorithm is presented in full detail in $[8,9]$.

\section{MULTIMODAL DATA COLLECTED}

Data are collected from children playing with a physical interactive game. The game, called Bug-Smasher, is designed using the Playware playground (interactive tiles) platform [4] in the study presented here. (The reader is referred to [9] for more details on Bug-Smasher). Seventy six children participated in the game survey experiment where each subject played a set of 90 second games in which their interaction data with the playground and specific physiological signals were recorded. Each subject was asked to express an entertainment preference for the two games she played (i.e. which one of the two games was more fun?). The two modalities of data consist of 71 , in total, statistical features extracted from gameplay interaction and physiological signals as outlined below.

\subsection{Gameplay Interaction}

Pressed tile events are recorded in real-time and a selection of nine player interaction features are calculated for each child. These include the child's score, the number of interactions with the game platform, the average and the variance of the response times, the average and the variance of the distance between pressed tiles and opponents appearing on the game; the average and the variance of the pressure on the tiles, and the entropy of the tiles that the child visited. The complete set of features also includes the quantitative controllable game features of game speed and opponent spatial diversity.

\subsection{Physiological Signals}

Physiology is the second modality investigated here. The physiological signals of heart rate (HR), blood volume pulse (BVP) and skin conductance (SC) are recorded while children play with the Playware games. This section lists the statistical features extracted from those three physiological signals. Some features are extracted for all signals while some are signal-dependent as seen in the list below. The choice of those specific statistical features is made in order to cover a decent amount of the HR, BVP and SC signal dynamics $[6,8]$.

All signals Average, standard deviation, maximum, minimum, the difference between maximum and minimum, correlation coefficient between raw recordings and time, autocorrelation, time when maximum and minimum occurred and the difference between them.

HR Initial and last HR recording, and the approximate entropy $\left(A p E n_{h}\right)$ of the signal — see [9] for further details on $A p E n$.

BVP Average inter-beat amplitude, mean of the first and second differences of the raw BVP, mean of the absolute values of the first and second differences of the BVP signal [6]. Moreover, given the inter-beat (RR) 
time intervals of the BVP signal a number of Heart Rate Variability (HRV) parameters are computed within the HRV time and frequency domain following the clinical study reported in [2]. HRV parameters include the standard deviation of RR intervals, the fraction of $\mathrm{RR}$ intervals that differ by more than $50 \mathrm{msec}$ from the previous $R R$ interval, the root-mean-square of successive differences of RR intervals and the frequency band energy values derived from power spectra obtained using discrete Fourier transformation on the RR intervals.

SC All extracted features used for the HR signal. Additional features include the mean of the first and second differences of the raw SC and the mean of the absolute values of the first and second differences of the SC signal.

\section{FEATURE SELECTION}

Feature selection is utilized to find the feature subset that yields that most accurate user model and save computational effort of exhaustive search on all possible feature combinations. The quality of the predictive model constructed by the preference learning outlined above depends critically on the set of input data features chosen. Using the extracted features described above, Sequential Forward Selection (SFS) and Perceptron Feature Selection (PFS) are applied and compared.

The SFS method is a bottom-up search procedure where one feature is added at a time to the current feature set. The feature to be added is selected from the subset of the remaining features such that the new feature set generates the maximum value of the performance function over all candidate features for addition. The SFS method is used since it has been successfully applied to a wide variety of feature selection problems, yielding high performance values with minimal feature subsets $[8,11]$

The second method we investigate is perceptron pruning (a variant of sequential backward selection) as a methodology for selecting appropriate feature subsets. Our algorithm which is similar to [5] is adjusted to match preference learning problems. Thus, the perceptron used employs the sigmoid activation function in a single output neuron. The ANN's initial input vector consists of all features extracted $\mathcal{F}$ (71 in this paper). The perceptron feature selection (PFS) procedure is as follows: (a) Use artificial evolution to train the perceptron on the pairwise preferences (performance of the perceptron is evaluated through 3 -fold cross-validation); (b) eliminate all features $\mathcal{F}^{\prime}$ whose corresponding absolute connection weight values are smaller than $E\{|\mathbf{w}|\}-\sigma\{|\mathbf{w}|\}$, where $\mathbf{w}$ is the connection weight vector; (c) if $\mathcal{F}^{\prime}=\emptyset$ continue to (d), otherwise use the remaining features and go to (a); (d) evaluate all feature subsets obtained using the neuro-evolution preference learning approach presented in Section 3.1.

Note that both methods are incomplete. Neither is guaranteed to find the optimal feature set since neither searches all possible combinations (they are each a variant of hillclimbing). To evaluate the performance of each input feature subset, the available data is randomly divided into thirds and training and validation data sets consisting of $2 / 3$ and $1 / 3$ of the data respectively are assembled. The performance of each user model is measured through the average classification accuracy of the model in three independent runs using the leave-one-out cross-validation technique on the three possible independent training and validation data sets. Since we are interested in the minimal feature subset that yields the highest performance we terminate the SFS selection procedure when an added feature yields equal or lower validation performance to the performance obtained without it. On the same basis, we store all feature subsets selected by PFS and explore the highest performing subset starting with the smallest feature subset generated.

\section{BEST MODEL OBTAINED}

Preference learning combined with the two feature selection methods is applied on the data collected. It was empirically determined that ANN architectures with 10 hidden neurons, are capable of successfully obtaining solutions of high fitness. This was determined by considering the performance of ANN architectures with up to two hidden layers containing up to 30 hidden neurons each.

\subsection{Feature Selection Method Comparison}

Comparing the two feature selection methods the SFS method generates feature subsets that yield higher validation performance than feature subsets generated by PFS, given the same number of features (see Figure 2). Moreover, PFS highest performing feature subset (76.54\%) consists of 8 features whereas the corresponding subset for SFS $(83.95 \%)$ consists of only 3 features. (Note that, the performance of randomly generated networks lies between $48 \%$ and $52 \%$ for all input vectors chosen by the two feature selection schemes.) On the other hand, out of $C_{3}^{71}=57155$ combinations available SFS tries $71+70+69=210$ combinations of features. The corresponding number of combination trials for PFS is 15. The difference of the searching strategy reflects on their performance difference. Even though PFS is computationally preferred, SFS manages to find a much higher performing subset with reasonable computational effort. An effort cost analysis [10] would reveal the exact relationship between efficacy and effort but we judge that such an analysis is out of the scope of this paper. Given that our priority is to generate the most accurate user model out of the multimodal data collected, we accept the SFS solution thereafter.

\subsection{Comparison Against Unimodal Models}

Obtained best performance $(83.95 \%$; standard deviation equals 4.27 ) while satisfactory it shows the difficulty in distinguishing multimodal data between games in terms of the reported preferences of entertainment. The reported complexity of classifying emotions [6], the augmented signal noise recorded during physical play and the binomially-distributed probability of this performance to occur at random (0.0057) demonstrate the efficacy of the model generated.

User models built on the same set of data have already been reported; however, these models have been constructed on features extracted from single-modalities. Specifically, studies on constructing ANN user models on the interactiongame data (extracted from time and pressure events on the tiles) using SFS have generated models yielding $82.22 \%$ of classification accuracy [11]. Likewise, user models built solely on physiological data (HR, BVP and SC) of subjects generate an accuracy of $79.76 \%$ [8]. Table 1 presents the comparative analysis of model performance across data modalities. The unified set of features on which the multimodal user 


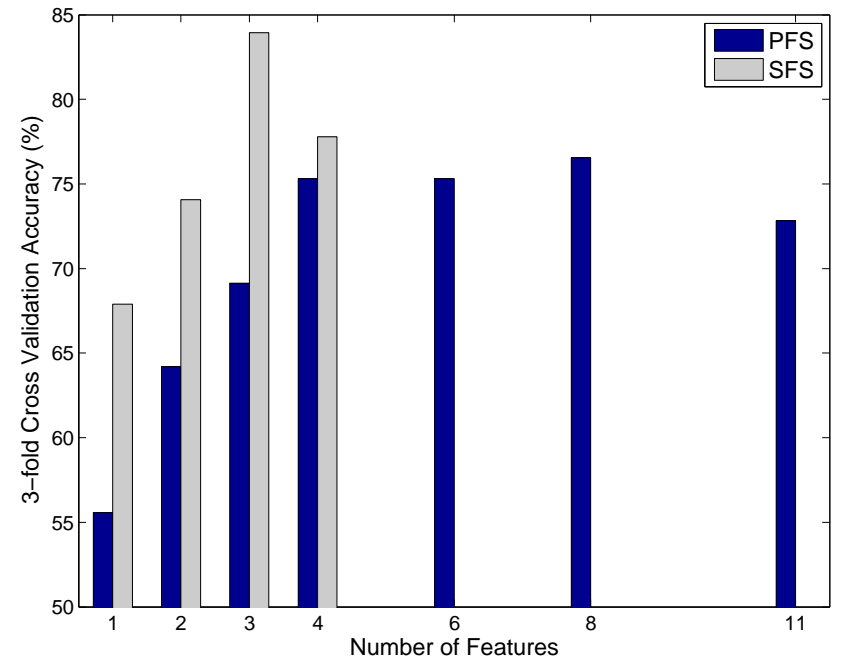

Figure 2: Performance vs. selected feature subset size.

model is built performs higher $(83.95 \%)$ with only three features selected providing indications for the benefits of multimodality when constructing user models of reported emotional experiences (i.e. player satisfaction in this paper).

The controllable feature of opponent spatial diversity, $H$, the average blood volume pulse, $E\{b\}$, and the energy of the high frequency $([0.15,0.4] \mathrm{Hz})$ band of heart rate variability (derived from power spectral analysis), HF, [2] are the three features selected by SFS. Average blood volume pulse and $\mathrm{HF}$ are indicators of sympathetic arousal and emotional stress $[7,2]$, respectively, while $H$ is an estimate of the player's curiosity generated from the game investigated. Further analysis of the multimodal model will be required to reveal the exact relationship between the selected features and reported fun and connect that relationship to established theoretical frameworks of emotion.

Table 1: Classification accuracy (\%) over different data modalities. Evolving ANN using SFS for selecting the ANN input vector.

\begin{tabular}{c||c|c|c}
\hline \hline No. of Features & Gameplay & Physiology & Multimodal \\
\hline 1 & 62.22 & 66.67 & 67.90 \\
2 & 67.77 & 71.43 & 74.07 \\
3 & 68.88 & 79.76 & 83.95 \\
4 & 82.22 & 75.00 & 77.78 \\
\hline \hline
\end{tabular}

\section{CONCLUSIONS}

This paper demonstrated the advantages of using data extracted from multiple modalities and automatic feature selection for building models of user preference. The highest performance obtained $(83.95 \%)$ outperforms the accuracy of both the model built solely on physiological data $(79.76 \%)$ and the model built solely on gameplay interaction data $(82.22 \%)$. Automatic feature selection chooses only 3 out of the 71 , in total, features which feed the highest performing ANN model: the controllable feature of opponent spatial diversity, $H$, the average blood volume pulse, $E\{b\}$, and the energy of the high frequency band of heart rate variability, HF. A deeper analysis on the generated ANN model will be required to expose the interplay between the three selected features and reported fun.

In the comparison between the two different features selection schemes utilized, results show the benefits of using sequential forward selection over perceptron feature selection for choosing the input vector of the model. SFS constitutes a good balance between performance and computational effort. The experimental methodology proposed for quantitatively modeling preferences of players is applicable to any multimodal interactive system for predicting preferences of users given individual characteristics of their interaction with the system.

\section{REFERENCES}

[1] J. Doyle. Prospects for preferences. Computational Intelligence, 20(2):111-136, May 2004.

[2] J. J. Goldberger, S. Challapalli, R. Tung, M. A. Parker, and A. H. Kadish. Relationship of heart rate variability to parasympathetic effect. Circulation, 103:1977-1983, 2001.

[3] J. H. Holland. Adaptation in Natural and Artificial Systems. University of Michigan Press, Ann Arbor, MI, 1975.

[4] H. H. Lund, T. Klitbo, and C. Jessen. Playware technology for physically activating play. Artifical Life and Robotics Journal, 9(4):165-174, 2005.

[5] M. Mejia-Lavalle and G. Arroyo-Figueroa. Power System Database Feature Selection Using a Relaxed Perceptron Paradigm. In Proceedings of 5th Mexican International Conference on Artificial Intelligence, LNCS, pages 522-531. Springer Berlin/Heidelberg, 2006.

[6] R. W. Picard, E. Vyzas, and J. Healey. Toward Machine Emotional Intelligence: Analysis of Affective Physiological State. IEEE Trans. Pattern Anal. Mach. Intell., 23(10):1175-1191, 2001.

[7] D. W. Rowe, J. Sibert, and D. Irwin. Heart Rate Variability: Indicator of User State as an aid to Human-Computer Interaction. In Proceedings of Conference on Human Factors in Computing Systems, pages 480-487, 1998.

[8] G. N. Yannakakis and J. Hallam. Entertainment Modeling through Physiology in Physical Play. International Journal of Human-Computer Studies, 66:741-755, October 2008.

[9] G. N. Yannakakis, J. Hallam, and H. H. Lund. Entertainment Capture through Heart Rate Activity in Physical Interactive Playgrounds. User Modeling and User-Adapted Interaction, Special Issue: Affective Modeling and Adaptation, 18(1-2):207-243, February 2008.

[10] G. N. Yannakakis, J. Levine, and J. Hallam. Emerging Cooperation with Minimal Effort. Rewarding over Mimicking. IEEE Transactions on Evolutionary Computation, 11(3):382-396, June 2007.

[11] G. N. Yannakakis, M. Maragoudakis, and J. Hallam. Preference Learning for Cognitive Modeling: A Case Study on Entertainment Preferences. IEEE Systems, Man and Cybernetics; Part A: Systems and Humans, 2009. (to appear). 